\title{
Methods and Models of a Multifunctional System Support for Decision Making for Differential Diagnosis of Diseases
}

\author{
T.S.Safarov ${ }^{1}$,Sh.U. Urakov ${ }^{2}$, G.T.Safarova ${ }^{3}$, R.A.Sobirov ${ }^{4}$ \\ ${ }^{1}$ Samarkand branch of Tashkent University of Information Technology named after Muhammad al-Khorezmi, \\ Uzbekistan,safarov.t1953@gmail.com \\ ${ }^{2}$ Samarkand State Medical Institute, Uzbekistan, shokirurakov74@mail.ru \\ ${ }^{3}$ Samarkand branch of Tashkent University of Information Technology named after Muhammad al-Khorezmi, Uzbekistan, \\ st_gulnoza@mail.ru \\ ${ }^{4}$ Samarkand branch of Tashkent University of Information Technology named after Muhammad al-Khorezmi, Uzbekistan, \\ sruzimboy44@gmail.com
}

\begin{abstract}
The article discusses the development of a multifunctional system for the support of medical decision-making for the differential diagnosis of the disease based on the modular principle. It is known that due to the complexity of processing large volumes of information with a large number of decision rules in the knowledge base (KB), the decision-making process is complicated. In connection with this, usually an expert system with the aim of increasing the accuracy of diagnostic solutions isdeveloped as one functional expert systems (ES) for a narrow class of diseases. A multifunctional medical decision support systems (MDSS) will be designed to solve the problems of differential diagnosis of not one but many classes of diseases, which complicates the decision-making process. This paper discusses the development of multifunctional MDSS. Facilitation of the diagnostic decision-making process is carried out using the modular principle. Each module has a pile of knowledge bases, which has a hierarchical structure designed for phased reasoning. The software part of the ES consists of control programs and several modules (subprograms). Each module is designed to solve the differential diagnosis of one narrow class of the disease. The implementation of this system is considered for examples of the class of disease "Myocardial infarction" and "Headache". By increasing the number of modules in the $\mathrm{KB}$, you can expand the versatility of this diagnostic system.
\end{abstract}

Key words: Diagnostic, system, intellect, decision, multistage, expert system, module, rules, knowledge base, data base.

\section{INTRODUCTION}

Currently, in modern medicine, the advantage of modern information technology is widely used, which improves the quality of service, reduces examination time, increases diagnostic accuracy, and also provides the possibility of long-term storage of patient information in digital form. Recently, in medical practice and the educational system in the healthcare sector, intelligent decision support systems (DSS) in the form of ES are widely used. ES function with the help of expert knowledge and operational data obtained from the patient. At the same time, the knowledge of experts in a formalized form is stored in the knowledge base, and the data obtained from the patient by traditional, laboratory, instrumental and computer (TLIC) methods are in the database (DB). The TLIC method allows the completeness of information in the database.

Currently, the application of a systematic approach in the process of making a medical diagnostic decision gives an effective result with direct computer support for medical activities in medical institutions that allow you to quickly solve complex diagnostic and treatment situational tasks. This is based on a logical approach to the processing of medical data obtained by TLIC methods.

A review of existing literature [1-5] shows that the existing diagnostic decision support systems are being developed as ES for a narrow class of diseases.

The results of the analysis of existing works show that the issues of increasing the multifunctionality of MDSS, functioning as one subsystem of medical information systems (MIS), have not been sufficiently studied.

Because of the above, the goal of this work is to develop methods and models of multifunctional MDSS, for the organization of automated workplaces of a doctor common practice.

\section{METHODS AND MATERIALS}

In general terms, the term "Decision Support System (DSS)" refers to a computer system that, through the collection and analysis of information, can influence decision-making processes in various fields of human activity. In health care, such decisions are referred to as the amended term "medical decision support systems (MDSS)." 
MDSS - a medical information system designed to help doctors and other medical professionals in working with tasks related to clinical decision making. The development and implementation of MDSS into practice belong to the most important areas of the development of artificial intelligence in medicine.

MDSS, among others, has one functional characteristic, undeniably associated with improving the quality of work of practicing doctors, these are automatic electronic prompts instead of the need to activate the system by the user [6-10].

The main goal of modern HCWS is to help doctors at the place of medical care. This means that doctors are interacting with HRPVR to get support in analyzing the information and establishing a diagnosis based on the available data regarding the patient.

For complex modeling of MIS, you can use the complex model [11].

To create MDSS, various modeling methods can be used, in particular, methods of correlation and regression analysis [12], methods of probability theory and mathematical statistics [13,14], logical reasoning models $[15,16]$ and other methods, for example[17]. It is clear that due to the complexity of processing large amounts of information with a large number of decision rules located in the knowledge base, the decision-making process is complicated. In this regard, usually MDSS with the aim of increasing the accuracy of diagnostic solutions are developed in the form of one functional ES for a narrow class of diseases. Here, under one function we mean the possibility of using differential diagnosis of one narrow class of the disease. A multifunctional MDSS will be designed to solve the problems of differential diagnosis of not one, but many classes of diseases.

For the effective functioning and multifunctionality of the MDSSKB must be organized on a modular basis. Each module in the knowledge base will contain a set of decision rules for a specific homogeneous class of the disease (HCD). At the same time, MDSS consists of several subprograms intended for differential diagnosis of a certain class of diseases. Communication and control over the functioning of the model is carried out by the control program using operational data and data in the database. To collect operational data and data to fill the database, it is recommended to use the TLIC method, which ensures the reliability and completeness of information.

Thus, the general structure of multifunctional MDSS contains control programs and several modules (subprograms) intended for differential diagnosis of a narrow class of the disease. Increasing the number of modules increase the versatility of multifunctional ES [18].

For the functioning of control programs as operational data, the code of a homogeneous class of the disease is first introduced. To determine the class code of the disease, an international disease classifier is used.
Next, the control program on the basis of the class code of the disease selects the appropriate module to solve the differential diagnosis of the disease. Each module has its own database and database. KB contain all the rules, in the form of separate models for each class of the disease. KB has a hierarchical structure [19]. All rules are developed according to the knowledge of experts or according to evidence-based evidence-based medicine. When developing the rules for the knowledge base, production modeling methods are used.

\section{RESEARCH RESULTS}

The proposed diagnostic decision support system has the structure proposed in Figure 1.

MDSS function as a subsystem in the composition of MIS. As shown in Figure 1, the SPPVR contains all of the following subsystems:

- DDSS (diagnostic decision support system);

- SSAMP (support system for the adoption of medical prescriptions).

In this way, the MDSS is developed by integrating the DDSS and the SSAMP. Integrated MDSS is more effective for a general practitioner.

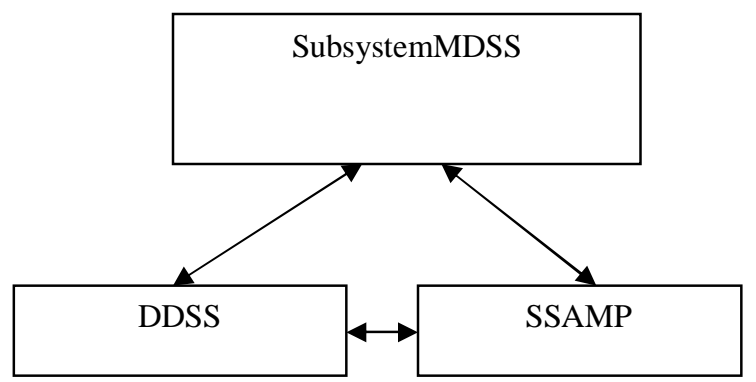

Figure 1: Structures of the subsystem MDSS (two-way arrow means informational relationships).

The implementation of this MDSS subsystem is based on the $\mathrm{KB}$, which contains the following two modules developed according to the hierarchical principle [19]. The first module uses the following set of rules for the differential diagnosis of myocardial infarction:

Stage I. The group of rules of knowledge for the diagnosis of myocardial infarction by symptoms:

The rule of the IM 1.1. If $\{<$ heart rhythm disturbance>, $<$ increase in blood pressure $>,<$ Friction noise $>,\langle E C G$ changes $>\}$ then $\{$ Significant suspicion of myocardial infarction \{Recommended rule 1.2$\}$. The rule of the IM 1.2. If $\{<$ Pain in the region of the heart $>,<$ Fever $>$, $<$ Muffling of heart sounds $>$ \} then \{Diagnosis: myocardial infarction $\}$. Rule IM 1.3. If $\{<$ Rule 1$\rangle$ or $<$ Rule $2>$ is not satisfied $>\}$ then $\{$ Further investigation is required $\}$.

Stage II. Differential diagnosis of myocardial infarction. The rule is developed by changing ECGs that mimic myocardial infarction. The rule of the IM 2.1. If $\{<\mathrm{ST}$ segment elevation $>\}$ then $\{$ Diagnosis: pericarditis $\}$ 
\{Recommendation: Echo of the CG\}. The rule of the IM 2.2. If $\{<$ ST segment elevation>, <appearance of $Q$ waves $>$ then $\{$ Diagnosis: myocarditis \{Recommendation: Echo of the CG\}.

The rule of the IM 2.3. If $\{<$ Rise or depression of the ST segment $>$, <Nonspecific changes in the ST segment and $\mathrm{T}$ waves $>$ \} then \{Diagnosis: Exfoliating aortic aneurysm\} \{Recommendation: Echocardiography, chest CT, aortography $\}$. The rule of the IM 2.4. If $\{<A$ slight increase in the amplitude of the $\mathrm{R}$ waves from V1 to V6>, < a sudden change in the electrical axis of the heart>\} then \{Diagnosis: Pneumothorax \{Recommendation: Chest X-ray\}. The rule of MI 2.5. If $\{\langle$ ST segment elevation in leads II, III, aV F $\rangle$, <inversion of $\mathrm{T}$ waves in $\mathrm{V} 1-\mathrm{V} 3>\}$ then $\{$ Diagnosis: TELA $\} \quad$ Recommendation "Ventilation-perfusion scintography" $\}$. The rule of MI 2.6. If $\{<\mathrm{ST}$ segment elevation in departments II, III, and V F $>\}$ then

\{Diagnosis: Acute cholecystitis\} \{Abdominal ultrasound recommendation $\}$.

Stage III. Rule on the results of enzymes: Rule IM 3.1. If $\{<$ Increase in the activity of the CF fraction of KFK after 8-10 hours $>$, <return to normal after 48-72 hours, ><increase in the activity of the MV fraction of KFK reaches a peak after 24-36 hours> \} then \{Diagnosis: myocardial infarction\}.

Stage IV. The rule is based on the results of echocardiography. The rule of the IM 4.1. If $\{<$ Violations of local left ventricular contractility $\rangle$, <Thinning of the left ventricular wall>\} then \{Diagnosis: Postponed myocardial infarction .

The rule of the IM 4.2. If $\{<$ Normal left ventricular contractility> $\}$ then

$\{$ The diagnosis of myocardial infarction is excluded \}.

Stage V. According to the results of coronary antigraphy. Rule IM 5.1. If $\{<$ Violations of local left ventricular contractility>, <Thrombotic occlusion of the coronary artery>\} then \{Diagnosis: Myocardial infarction $\}$.

As a BZ 2-module, you can use the set of rules developed for a homogeneous class of the disease "Headache", which contains the following rules:

I Stage. Rule reasoning by symptom and character. GB rule 1.1. If $\{\langle$ Nausea $\rangle,\langle$ Vomiting $\rangle,\langle$ Ailment $\rangle$, $<$ Photophobia $>$, <Throbbing $>$ then \{Diagnosis: Simple migraine $\}\{$ Recommendation: Verification according to GB 2.7 rule $\}$. GB rule 1.2 . If $\{\langle$ Nausea $\rangle$, $<$ Vomiting>, <Malaise>, <Photophobia>, <Visual aura>, <Throbbing $>$ Then \{Diagnosis: Classical migraine $\{$ Recommendation: checks according to the rule GB 2.2$\}$. GB rule 1.3 . If $\{\langle$ Nausea $\rangle$, <Vomiting $\rangle$, $<$ Dull or throbbing $>\}$ Then \{Diagnosis: Facial migraine\} \{Recommendation: check according to the rule $2.3 \mathrm{~GB}\}$. GB rule 1.4. If $\{<$ Hyperemia of the face $>$, <Nasal congestion>, <Horner's syndrome>, $<$ Acute drilling $>$ Then \{Diagnosis: Horton's headache\} \{Recommendation: check according to the rule 2.4 GB\}. Rule GB 1.5. If $\{<$ Depression>, $<$ Anxiety>, <Shooting $>$ THAT TDiagnosis:
Psychogenic headache\} \{Recommendation: check according to the rule of GB 2.5\}. GB rule 1.6. If $\{<$ Trigger zones are detected>, <Firing $>$ Then \{Diagnosis: Trigeminal neuralgia\} \{Recommendation: check according to the rule $2.6 \mathrm{~GB}\}$. Rule GB 1.7. If $\{\langle$ Depression is sometimes psychosis $\rangle,\langle$ Dumb $\rangle$ \} Then \{Diagnosis: Atypical facial pain \{Recommendation: check according to the rule of GB 2.7\}. GB rule 1.8. If \{<Nasal discharge $\rangle$, <Dull or sharp $>$ \} Then \{Diagnosis: Headache with sinusitis \{Recommendation: check according to the rule of GB 2.8.\}.

Stage II. Rule II of the stage is developed according to the characteristic features of the localization "Duration of an attack and periodicity”. GB Rules 2.1. If \{<One or two sided $>,<6$ to 48 hours $>$, $<$ Sporadic seizures (up to several times a month) $>\}$ then $\{$ Diagnosis: Simple migraine $\}$. Rule GB 2.2. If $\{\langle$ Unilateral $\rangle$, <from 3 to 12 hours $>$, <Sporodic seizures (up to several times a month) $>$ \} then \{Diagnosis: Classical migraine . GB rule 2.3. If $\{\langle$ Unilateral, in the lower half of the face $\rangle$, <from 6 to 48 hours>, <Sporodic attacks> $\}$ then \{Diagnosis: Facial migraine\}. GB rule 2.4. If $\{$ <unilateral (mainly in the area of the orbit)>, <from 15 to 120 minutes>, <Alternating periods of daily attacks and prolonged remissions $>$ \} then \{Diagnosis: Horton's headache $\}$. GB rule 2.5 . If $\{<$ Diffuse bilateral>, <often constant $>\}$ then \{Diagnosis: Psychogenic headache $\}$. GB rule 2.6. If $\{<\mathrm{In}$ the trigeminal nerve innervation zone $\rangle$, <briefly (from 15 to 60 seconds)>, <many times a day> $\}$ then \{Diagnosis:Trigeminal neuralgia\}. Rule GB 2.7. If $\{<$ One or two sided $>$, <often constant $>\}$ then \{Diagnosis: Atypical facial pain\}.GB rule 2.8. If \{ $<$ One or two-sided in the paranasal sinus $\rangle$, $<$ variable>, <Sporadic or constant $>$ \} then \{Diagnosis: Headache with sinusitis $\}$.

The SSAMP subsystem is organized according to the principle of quasi-assorted selection of effective treatment options.

\section{CONCLUSION}

Recommended multifunctional MDSS operates through the joint implementation of the subsystem DDSS and SSAMP. All modules included in the MDSS function with the corresponding knowledge base and database under the control of the "Control Programs" and gives recommendations for diagnosis and treatment.

The number of modules determines the number of functions MDSS. Such types of MDSS are of great practical importance in medical institutions where there are not enough experienced specialists. The widespread introduction of simple and intuitive systems, implemented, for example, in the form of technologically simple systems and ready for widespread use, are effective in self-learning when used by doctors in general healthcare practice or by students in the educational process. 


\section{REFERENCES}

[1] Litvin, A.A., Litvin V.A.System of support of decision-making in surgery //News of surgery.- 2014. \# 22(1).P.96-100 [in Russian]

https://doi.org/10.18484/2305-0047.2014.1.96

[2] Nazarenko G.I., Klejmanova E.B., ZhujkovM.Ju., Pajushik, Novakova A.I., Kashevskaja O.P., Jashina L.P. System of automation of clinical guidelines and audit of treatment // Medical information system]2014.\#2. P.23-32 [ in Russian]

[3]NajdanovCh.A. The system of support of decisionmaking to prevent risk of occurrence of critical conditions // Almanac of modern science and education .-2015.\#8 (98).P. 92-95 [ in Russian]

[4]Ravodin R.A. Intelligent system for supporting the adoption of medical decisions in dermatovenereology // Problems of medical mycology.2014.T.16. № 3. P. 5965.

[5]Zaripova G.R.,Bogdanova J.A., KataevV.A.Actual models of support systems for medical decisionmaking in surgical practice // INTERNATIONAL RESEARCH JOURNAL. № 11 (53), Part 3, 2016.Ekaternburg.p. 137-142

DOI: https://doi.org/10.18454/IRJ.2016.53.218.

[6] Moja, L; Kwag, KH; Lytras, T; Bertizzolo, L; Brandt, L; Pecoraro, V; Rigon, G; Vaona, A; Ruggiero, F; Mangia, M; Iorio, A; Kunnamo, I; Bonovas, S. Effectiveness of computerized decision support systems linked to electronic health records: a systematic review and metaanalysis (англ.) // American Journal of Public Health (англ.)русск. : journal. 2014. December (vol. 104, no. 12). P. e12-22

doi:10.2105/ajph.2014.302164. PMID 25322302.

[7] Garg A.X., Adhikari N.K., McDonald H., RosasArellano M.P., Devereaux P.J., Beyene J. et al. Effects of computerized clinical decision support systems on practitioner performance and patient outcomes: a systematic review (англ.) // JAMA : journal. 2005. Vol. 293, no. 10. P. 1223-1238.

doi:10.1001/jama.293.10.1223. PMID 15755945.

[8]Kensaku Kawamoto; Caitlin A Houlihan; E Andrew

Balas; David F Lobach. Improving clinical practice using clinical decision support systems: a systematic review of trials to identify features critical to success (англ.) // ВMJ : journal. 2005. Vol. 330, no. 7494. - P. 765.

doi:10.1136/bmj.38398.500764.8F. PMID 15767266.

[9] Black, A.D.; Car, J.; Pagliari, C.; Anandan, C.; Cresswell, K.; Bokun, T.; McKinstry, B.; Procter, R.; Majeed, A.; Sheikh, A. The impact of ehealth on the quality and safety of health care: A systematic overview (англ.) // PLoS Medicine (англ.)русск. : journal 2011.18 January (vol. 8, no. 1). P. e1000387. doi:10.1371/journal.pmed.1000387.

PMID 21267058.

[10] Nachtigall, I; Tafelski, S; Deja, M; Halle, E; Grebe, M C; Tamarkin, A; Rothbart, A; Unrig, A; Meyer, E; Musial-Bright, L; Wernecke, K D; Spies, $C$. Long-term effect of computer-assisted decision support for antibiotic treatment in critically ill patients: a prospective 'before/after' cohort study (англ.) // ВMJ Open (англ.)русск. : journal. — 2014. — 22 December (vol. 4, no. 12). P. e005370. doi:10.1136/bmjopen2014-005370. — PMID 25534209.

[11]UrakovSh.U., Safarov T.S. Complex model of acceptance of diagnostic decisions of hybrid intellectual support systems. // Ninth World Conference on Intelligent Systems for Industrial Automation (WCIS-2016)/. -Tashkent, Uzbekistan. 2016, p. 169-173.

[12]Safarov T.S.., Turakulov, S.X., Nabiyeva, I.S., Nabiyeva, S.S. Efficiency medical information systems in diagnostics.ISJ Theoretical \& Applied Science, 11 (79), 301-305. Year: 2019 Issue: 11 Volume: 79 ,Published: 20.11.2019 http://T-Science.org.(Doi: https://dx.doi.org/10.15863/TAS).

[13]Zhmudyak M.L., Povaliikhin A.N., Strebukov A.V. et al. Diagnosis of diseases by methods of probability theory // Alt. state those. un-t them. I.I. Polzunova. - Barnaul: AltSTU Publishing House, 2006. $-168 \mathrm{p}$.

[14]Safarov T.S., UrakovSh.U. A matrix probabilistic model for creating counseling systems for medical diagnostics. // Materials of the Republican Scientific Conference "New Theorems of Young Mathematicians", Namangan, 2009, p. 32-34.

[15]Safarov T.S., UrakovSh.U., Rustamov A.A., TurakulovSh.Kh. The modular principle of developing a multifunctional expert system for the differential diagnosis of diseases. Electronic Journal: Questions of Science and Education. Moscow, №28, 2019, c.103109. https://scientificpublication.ru/h/arkhiv-zhurnalavoprosy-nauki-i-obrazovaniya.html

[16] Sri Hari Nallamala1, Dr. Pragnyaban Mishra2, Dr. SuvarnaVaniKoneru.Qualitative Metrics on Breast Cancer Diagnosis with Neuro Fuzzy Inference Systems.International Journal of Advanced Trends in Computer Science and Engineering, Volume 8, No.2, March - April 2019, pp.260-264, doi.org/10.30534/ijatcse/2019/26822019.

[17] Oguntimilehin A.1, Babalolabemisola. O2, Olatunji K.A. A Clinical Diagnostic Model Based on Supervised Learning. International Journal of Advanced Trends in Computer Science and Engineering, 8(3), May - June 2019, pp.949 953doi.org/10.30534/ijatcse/2019/94832019

[18]Safarov T.S., UrakovSh.U.About one method of expanding the versatility of diagnostic systems. // Uzbek journal "Problems of Informatics and Energy", Tashkent, 2014, No. 5, p. 88-94.

[19]Safarov T.S., UrakovSh.U. Organization of the hierarchical structure of the knowledge base and their application in medical diagnostics. // Uzbek journal "Problems of Informatics and Energy", Tashkent, 2011, No. 6, p. 45-48. 